\title{
Association between change in heart rate over years and life span: Paris Prospective 1, Whitehall 1, and Framingham studies
}

\section{Bamba Gaye}

Hôpital Européen Georges-Pompidou

Eugenie Valentin

Paris Cardiovascular Research Center

Vanessa Xanthakis

Boston University

Marie-Cecile Perier

Paris Cardiovascular Research Center

David Celermajer

University of Sydney

Martin Shipley

University College London

Eloi Marijon

Hôpital Européen Georges-Pompidou

Rebecca Song

Boston University

Jean-Philippe Empana

Paris Cardiovascular Research Center

Vasan Ramachandran

Boston University

Xavier Jouven ( $\nabla$ xavier.jouven@inserm.fr)

Hôpital Européen Georges-Pompidou

\section{Research Article}

Keywords:

Posted Date: March 7th, 2022

DOI: https://doi.org/10.21203/rs.3.rs-1302298/v1 
License: (c) (i) This work is licensed under a Creative Commons Attribution 4.0 International License. Read Full License 


\section{Abstract}

We examined the association between resting heart rate and life span using linear regression in the Paris Prospective Study I, the Whitehall I Study, and the Framingham Heart Study. We used Cox proportional hazards regression to relate change in heart rate over years to mortality risk. We confirmed the inverse relation between resting heart rate and life span, observed a shift to the right (an increase) in life span over the past 250 years in humans compared to other mammals, and observed that higher resting heart rate was associated with higher risk of mortality after adjustment for standard risk factors.

\section{Introduction}

Across mammalian species, larger body size is associated with lower heart rate and a longer life span. ${ }^{1-3}$ Heart rate, a measure of the frequency of the cardiac cycle, reflects health of the cardiovascular system, metabolic rate, and activity of the autonomic nervous system. Evolution has shaped optimal body size and heart rate in various species across millennia such that there is a neat inverse association between the two. The astonishing increase in human life span in the past two centuries raises the question of whether the human species is an outlier in the inverse heart rate-life span association. ${ }^{4}$ Improvements in health and social care underlie improvements in life span but there are concerns that the omnipresent obesogenic environment may undermine further progress. In the original Framingham Heart Study cohort of 5070 subjects, a greater death rate was observed in individuals with faster heart rate, who were free of cardiovascular disease at the entry of the study and followed for 30 years. ${ }^{5}$ However, to our best knowledge, whether change in resting heart rate is related to lifespan has not been yet explored. This would be an important step to increase our knowledge on a potential causal relationship between HR and lifespan, which may have clinical and public health implications.

To understand the association between heart rate and life span, we had 4 aims: 1) to confirm the inverse association between resting heart rate and lifespan across mammalian species; 2) to confirm the inverse association between resting heart rate and lifespan within human populations; 3 ) to assess if changes in human life span over the past 250 years "shifted" modern humans off this heart-rate versus life span relationship for all other mammals, including pre-industrial humans; and 4) to look for any association between increase in heart rate (over a 5-year period in the Paris Prospective Study I and 8-years period in the Framingham Study) and risk of mortality in modern humans.

\section{Online Methods}

\section{Data}

Historical data on human life span: The French National Institute of Demographic Studies has collected comprehensive data on life span in France from 1750, at 50 year intervals. ${ }^{13}$ Data on heart rate was not available here, we assumed that mean heart rate was 80 throughout this period. 
Paris Prospective Study I: This study is based on 7976 participants, aged 42 to 53 years, recruited to the study from 1967 to 1972 with a $93.4 \%$ response rate. ${ }^{14,15}$ Briefly, oral informed consent was given by each participant, and the research protocol was approved by the institutional board, Commission Nationale Informatique et Liberté. Clinical examination consisted of electrocardiograms, physical examination, and collection of blood samples for laboratory tests; participants also responded to a questionnaire administered by trained interviewers. Resting heart rate was determined by measurement of the radial pulse during a one-minute recording, after a five-minute rest in supine position. The covariates assessed were age, smoking (mean consumption g/days over the 5 last years), diabetes (selfreport of history of diabetes, whether treated), body mass index, current sport, systolic blood pressure, total cholesterol. Participants were invited back for a clinical examination each year for the following 4 years using the same procedure; a total of 5589 provided a heart rate at the final follow-up (five years from the baseline follow-up).

Mortality data were collected from medical records in hospital departments or general practitioners and death certificates. The end of the follow-up period was 1st January, 1994. The vital status could not be determined for 355 subjects (4.6\%). The baseline characteristics of those 355 subjects lost to follow up and of those who did not complete the final follow-up examinations (five years from the baseline followup) were not different from the remaining 5589 men studied in the present analysis. Vital status was followed on all subjects until the last recorded survey in 1994 (25 years of average follow up), through the administrative department in charge of the study population until retirement age and then thereafter via the French national death registry.

Whitehall I Study: Data were collected from 19,019 male London-based government employees aged 40 to 69 at screening between 1967 and 1970, with a 77\% response rate. Participants provided informed written consent and research ethics approval was obtained from the National Health Service London Harrow Research Ethics Committee. Screening involved the completion of a study questionnaire and participation in a medical examination, both of which have been described in detail elsewhere. ${ }^{16}$ Heart rate was measured in a random subset of 1263 men (mean age 52, SD 7 years) in whom radiologic heart volume was calculated. ${ }^{17}$ The covariates assessed in this age, smoking, diabetes, body mass index, current sport, systolic blood pressure, total cholesterol.

All cohort participants were traced using the National Health Service Central Registry for mortality for 40 years until 31st October 2008 by which time 977 men had died.

Framingham Heart Study: The final sample size for the current investigation consisted of 7300 participants (3299 men and 4001 women) from the Original (Examination 12, 1971-1974) and Offspring (Examination 1, 1971-1975) cohorts in the Framingham Heart Study after exclusion of participants $<20$ years of age $(n=245)$, with prevalent cardiovascular disease $(n=698)$, or a systolic blood pressure $>180$ $\mathrm{mm} \mathrm{Hg}(\mathrm{n}=198)$. Heart rate was measured by echocardiogram and data on covariates (age, sex, systolic blood pressure, current smoking, diabetes status, body mass index, physical activity index, and total cholesterol level) were drawn from the same wave. Written informed consent was provided by all 
participants and the study protocol was approved by the Boston University Medical Center Institutional Review Board.

Death were confirmed from death certificates and supporting information was obtained from hospital records. ${ }^{18}$ Participants were followed until 31 st December 2017, an average follow-up of 30.9 years for men and 32.8 for women.

\section{Data Availability Statement}

The datasets used and/or analysed during the current study are available from the corresponding author on reasonable request.

\section{Ethics Declaration}

All methods were carried out in accordance with relevant guidelines and regulations, informed consent was obtained from all subjects and/or their legal guardians.

\section{Statistical analysis}

Life span in all three cohorts was defined by age at death among participants who died over the follow-up period. For participants in the Paris Prospective and Whitehall studies who were alive at the end of the follow-up, life span was estimated based on national age and sex in the corresponding country.

Aim 1 and 2: As the sample size and number of deaths were larger in the Paris Prospective Study I and Framingham Study, heart rate was categorized into 5 groups: $\leq 60,60-70,70-80,80-90,>90 \mathrm{bpm}$. In the Whitehall I study smaller numbers led us to use 4 categories: $\leq 60,60-70,70-80,>80 \mathrm{bpm}$.

Participants with known or suspected cardiovascular disease or with any of the following conditions were excluded from the study: resting systolic blood pressure $>180 \mathrm{~mm} \mathrm{Hg}$, resting 12-lead standard electrocardiogram abnormality (Minnesota code). A linear regression was used to examine the association between resting heart rate and life span in each cohort study, first unadjusted and then adjusted for age, baseline body mass index, smoking, physical activity, diabetes, systolic blood pressure, and total cholesterol level.

Aim 3: The inverse linear relationship between logarithmic transformed heart rate and life span in mammalian species and humans was derived by Levine. ${ }^{1}$ We used data from the French National Institute of Demographic Studies to plot the point estimate every 50 years, for the period from 1750 (when earliest reliable records were available) until 2000. ${ }^{13}$

Aim 4: In the Paris Prospective Study I and Framingham Heart Study, multivariable Cox proportional hazards regression models were used to assess the change in heart rate (over a 5-year period in the Paris Prospective Study I and 8-years period in the Framingham Study) and risk of mortality. These analyses were adjusted for the covariates used in aim 2 along with resting heart rate at baseline. In the Paris Prospective Study, l, change in heart rate was defined as resting heart rate at the last follow-up visit minus resting heart rate at baseline. In the Framingham Heart Study, heart rate change (increase or decrease) 
was defined as resting heart rate at examination 16 (1979-1982) minus resting heart rate at examination 12 (1971-1974) for the Original cohort, and examination 2 (1979-1983) minus examination 1 for the Offspring cohort (1971-1975). A positive difference in heart rate indicated an increase in heart rate at the subsequent examination, while a negative difference indicated a decrease in heart rate.

SAS, Version 9.2 and 9.4 (Statistical Analysis System, Cary, N.C.) was used for analysis. P-values were 2sided.

\section{Results}

We investigated whether differences in resting heart rate within human populations are associated with life span using data from three population-based cohorts based in three different countries: the Paris Prospective Study I (France, Fig. 1a), the Whitehall I study (UK, Fig. 1b), and the Framingham Heart Study (USA, Fig. 1c-1d). In the Paris Prospective Study I, among 7976 healthy French men aged 42 to 53 years, recruited in the study from 1967 to 1972, 2387 deaths were recorded by 31st December 1993. There was an inverse linear relation between resting heart rate and life span, which remained significant after adjustment for potential confounders. Subjects with a resting heart rate over $90 \mathrm{bpm}$ had a life span of $70.27 \pm 12.78$ whereas subjects with a resting heart rate of less than $60 \mathrm{bpm}$ had a life span of $79.30 \pm$ 8.43 (HR mortality for 10 bpm difference: $1.25 ; 95 \% \mathrm{Cl}: 1.21$ to $1.30 ; \mathrm{p}<0.0001$ ) (Fig. 1a), corresponding to an average difference in life span of 9 years.

In the Whitehall I study, among the 1226 healthy British men aged $52 \pm 7$ years, recruited between 1967 and 1969, 977 had died at last follow up in 2008. Similar to the Paris Prospective Study I, an inverse resting heart rate-life span relation was also observed in both the unadjusted and adjusted models (Fig. 1b).

The analysis in the Framingham Heart Study extended the results to women. The participants were recruited from 1971 to 1975 . Among 4001 US women aged $47 \pm 16$ years, a total of 2409 deaths occurred over a mean follow-up time of 32.8 years. Among 3299 men aged $45 \pm 15$ years, a total of 2172 deaths occurred over a mean follow-up of 30.9 years. An inverse association was observed between resting heart rate and life span in both women and men. Women experienced longer longevity than men (5 years on average) for each category of resting heart rate (Figs. 1c-1d).

In addition, the known relation between heart rate and life span in mammalian species, juxtaposed with historical data on French men, and mean life span in the Paris Prospective Study I cohort study are shown in Fig. 2. The data show the progressive increase of life span to the right-hand side of Fig. 2 for men between the year 1750 ( 28 years) and 2000 ( 78 years), while the average heart rate remained constant.

Furthermore, we examined the association between change in resting heart rate over a 5-year and 8-year period and risk of mortality using data from the 5589 men in the Paris Prospective Study I as well as 3299 men and 4001 women in the Framingham Heart Study (Table 1). We observed a statistically 
significant association between increase in resting heart rate over a 5-year period and risk of mortality in French men from the Paris Prospective Study I (HR mortality per $10 \mathrm{bpm}$ increase over time: 1.20; 95\% Cl: 1.13 to 1.27) and over an 8-year period in American men and women from the Framingham Heart Study (HR: $1.13 ; 95 \% \mathrm{Cl}: 1.07$ to 1.19 for men and HR: $1.09 ; 95 \% \mathrm{Cl}: 1.04$ to 1.15 for women), after adjusting for classical risk factors and resting heart rate.

Table 1

Multivariable-adjusted hazard ratios for change in heart rate over 5-8 years and risk of mortality in the Paris Prospective Study I and the Framingham Heart Study

\begin{tabular}{|lllll|}
\hline Cohort & Sex & $\begin{array}{l}\text { Hazards Ratio } \\
\text { per } 10 \text { bpm increase }\end{array}$ & $\begin{array}{l}\text { 95\% Confidence } \\
\text { Interval }\end{array}$ & p-value \\
\hline PPSI & Male & 1.20 & $(1.13,1.27)$ & $<0.0001$ \\
\hline FHS & Female & 1.09 & $(1.04,1.15)$ & 0.0003 \\
\cline { 2 - 3 } & 1.13 & $(1.07,1.19)$ & $<0.0001$ \\
\hline
\end{tabular}

PPS I: A total of 1256 deaths among 5589 over a 25 years follow-up. Baseline follow up was at 1967.

FHS: A total of 1582 men and 1840 women had died over a mean follow-up of 30.9 years for men, and 32.8 years for women in 2461 men and 3068 women. Baseline follow up was between 1971 and 1975.

Heart rate change was determined using two measurements, the heart rate baseline and after 5 years in PPSI, and after 8 years in FHS

\& Subjects whose resting heart rate increased by 10 bpm over a 5-year period for PPS I and 8-year period for FHS, increased their risk of mortality $(p<0.001)$.

- Models are adjusted for age, smoking (mean consumption g/days 5 last years for PPS l; current smoking (yes/no) for FHS), diabetes, body mass index, physical activity, systolic blood pressure, and total blood cholesterol, and baseline heart rate.

\section{Discussion}

In this study, using three long-term historical and large cohorts from France, UK and USA, we reported 4 main findings. We confirmed the inverse relation between resting heart rate and life span, firstly, across mammalian species and secondly, within human populations. Thirdly, we observed a shift to the right (increase) in life span over the past 250 years in humans compared to mammals, corresponding with public health and medical advances that extended human life expectancy out of proportion to that of other mammals. Finally, we determined that men and women who increase their resting heart rate over a 5-year period in the Paris Prospective Study I and 8-years period in the Framingham Study, respectively, increase their risk of mortality.

The concept of total energy expenditure throughout the life span may be a part of the explanation of the association between heart rate and life span. Larger organisms lose heat as a function of their body 
surface area but generate heat as a function of their body mass. To conserve body temperature, smaller mammals with a higher surface area to mass ratio require an increased metabolic rate, which in turn determines heart rate. Thus, heart rate may be a "surrogate marker" for total energy expenditure, albeit much easier to measure accurately.

The same linear relation is observed between body size and longevity. Milnor proposed an explanation for the relation between heart rate, body size and aortic input impedance: the pulse wave length and arterial length are matched in a way that minimizes cardiac work. ${ }^{6}$ The lower maximal rate in larger animals probably reflects the longer time required for propagation and reflection of pulse wave as heart rate size increases. ${ }^{6}$ In our three studies, adjustments were performed for body mass index and did not alter the relation between heart rate and life span. In support of the key rate of energy expenditure as a determinant of mammalian life span, Rubner first noted in 1883 that total energy expenditure in a life span was approximately constant for 5 different species of domestic animal. ${ }^{7}$ In 1994, Azbel determined little variation in the total number of heart beats per lifetime in non-human mammals and found that energy consumption per body atom, or per heartbeat, was relatively constant, at approximately $10 \mathrm{O}_{2}$ molecules per atom per lifetime or approximately $10^{-8} \mathrm{O}_{2}$ molecules per atom per heartbeat. ${ }^{2}$ It is noteworthy, however, that fish and insects appear to have significantly fewer heart beats per life time than mammals (by approximately one order of magnitude), perhaps reflecting different environments and/or bioenergetic profiles.

Some experimental evidence has addressed the question of whether slowing heart rates in mammals might increase life span. Coburn et al found that slowing heart rate in mice by approximately $50 \%$ (using the pharmacologic agent digoxin) increased life span by approximately $23 \%$, however, this study was confounded by decreased body weight in the digoxin-treated mice. ${ }^{8}$ Beere et al reported that lowering heart rate in monkeys (by surgical interference with the cardiac pacemaker cells) led to slowed development of coronary atherosclerosis. ${ }^{9}$ In humans, inducing a slow heart rate has only been studied in the context of serious illness. In this setting, using beta adrenergic receptor blocker have been associated with decreased mortality risk in post-myocardial infarction patients with heart failure and a high resting heart rate. ${ }^{10,11}$ Furthermore, Fox et al reported that although reduction in heart rate with ivabradine, a pure bradycardic $I_{\mathrm{f}}$ channel blocker agent, has been shown to decrease the incidence of coronary artery disease outcomes in a subgroup of patients who have heart rates of $70 \mathrm{bpm}$ or higher, it has not been shown to improve cardiac outcomes in all patients with stable coronary artery diseases and leftventricular systolic dysfunction. ${ }^{12}$ However, the effects of a slowing heart rate on life span have not been studied in a healthy human population, and causality has not been addressed.

In addition to the biological plausibility argument summarized above, assessing the causality in epidemiology requires 3 steps. The first step is observational, and in the present study, we show independent association between higher heart rate and shorter life span. The second step is also observational and we newly demonstrate that individuals who increase their heart rate over a 5-year period in the Paris Prospective Study I and 8-years period in the Framingham Study, respectively, decrease 
their life span. Importantly, these results were obtained in populations from different countries and different risk factors distribution supporting the generalizability of the findings. Also, the differences in lifespan according to baseline or change in resting heart rate were meaningful in magnitude, which supports the clinical and public health relevance of our findings. The third step is interventional: when an intervention decreases heart rate, we observe an increase in life span in the long run. We acknowledge that this step will be much more difficult to demonstrate. Still, on the basis of the present findings, and given the simplicity of heart rate assessment, heart rate might be a target to monitor humans health and life span. Given the obesogenic environment of our societies, creating favourable environment to promote healthy behaviours that impact heart rate (i.e. physical activity) is more than ever a priority.

\section{Declarations}

\section{Author Contributions:}

BG and XJ had full access to all the data in the study and takes responsibility for the integrity of the data and the accuracy of the data analysis.

Study concept and design: XJ

Acquisition, analysis, or interpretation of data: BG, EV, VX, MCP, DSC, MS, EM, RJS, JPE, RSV, XJ

Drafting of the manuscript: $B G, X J$

Critical revision of the manuscript for important intellectual content: BG, EV, VX, MCP, DSC, MS, EM, RJS, JPE, RSV, XJ

Study supervision: XJ

\section{Additional Information}

Competing financial interests: The authors declare no competing financial interests.

Funding: None.

\section{References}

1. Levine, H. J. Rest heart rate and life expectancy. J. Am. Coll. Cardiol.30, 1104-1106 (1997).

2. Azbel MYa. Universal biological scaling and mortality. Proc. Natl. Acad. Sci. U. S. A.91, 12453-12457 (1994).

3. Zhang, G. Q. \& Zhang, W. Heart rate, lifespan, and mortality risk. Ageing Res. Rev.8, 52-60 (2009).

4. Oeppen, J. DEMOGRAPHY: Enhanced: Broken Limits to Life Expectancy. Science296, 1029-1031 (2002). 
5. Kannel, W. B., Kannel, C., Paffenbarger, R. S. \& Cupples, L. A. Heart rate and cardiovascular mortality: the Framingham Study. Am. Heart J.113, 1489-1494 (1987).

6. Milnor, W. R. Aortic wavelength as a determinant of the relation between heart rate and body size in mammals. Am. J. Physiol.-Regul. Integr. Comp. Physiol.237, R3-R6 (1979).

7. Rubner, M. Ueber den Einfluss der Körpergrösse auf Stoff- und Kraftwechsel. Z. Für Biol.19, 535-562 (1883).

8. Coburn, A. F., Grey, R. M. \& Rivera, S. M. Observations on the relation of heart rate, life span, weight and mineralization in the digoxin-treated A-J mouse. Johns Hopkins Med. J.128, 169-193 (1974).

9. Beere, P. A., Glagov, S. \& Zarins, C. K. Retarding effect of lowered heart rate on coronary atherosclerosis. Science226, 180-182 (1984).

10. Zhang, D., Shen, X. \& Qi, X. Resting heart rate and all-cause and cardiovascular mortality in the general population: a meta-analysis. Can. Med. Assoc. J.188, E53-E63 (2016).

11. Lechat, P. et al. Heart Rate and Cardiac Rhythm Relationships With Bisoprolol Benefit in Chronic Heart Failure in CIBIS II Trial. Circulation103, 1428-1433 (2001).

12. Fox, K., Ford, I., Steg, P. G., Tendera, M. \& Ferrari, R. Ivabradine for patients with stable coronary artery disease and left-ventricular systolic dysfunction (BEAUTIFUL): a randomised, double-blind, placebocontrolled trial. The Lancet372, 807-816 (2008).

13. Tables de mortalité françaises. Ined - Institut national d'études démographiques https://www.ined.fr/fr/publications/editions/donnees-statistiques/tables-de-mortalite-francaisescdrom/.

14. Jouven, X. et al. Heart-Rate Profile during Exercise as a Predictor of Sudden Death. N. Engl. J. Med.352, 1951-1958 (2005).

15. Ducimetiere, P., Cambien, F., Richard, JacquesL., Rakotovao, R. \& Claude, JeanR. CORONARY HEART DISEASE IN MIDDLE-AGED FRENCHMEN. The Lancet315, 1346-1350 (1980).

16. Reid, D. D. et al. CARDIORESPIRATORY DISEASE AND DIABETES AMONG MIDDLE-AGED MALE CIVIL SERVANTS. The Lancet303, 469-473 (1974).

17. Christie, D. Physical Correlates of Radiologic Heart Volume. Acta Radiol. Diagn. (Stockh.)19, 732-736 (1978).

18. Kannel, W. B., Wolf, P. A. \& Garrison, R. J. Framingham Study: An Epidemiological Investigation of Cardiovascular Disease. Section 34. Some Risk Factors Related to the Annual Incidence of Cardiovascular Disease and Death Using Pooled Repeated Biennial Measurements: Framingham Heart Study, 30-Year Follow-Up. Natl. Tech. Rep. Libr.- NTIS 474 (1987).

\section{Figures}


Figure Ia. Relation between resting heart rate (at study baseline) and life span in 7967 Wen from the Paris Prospective study
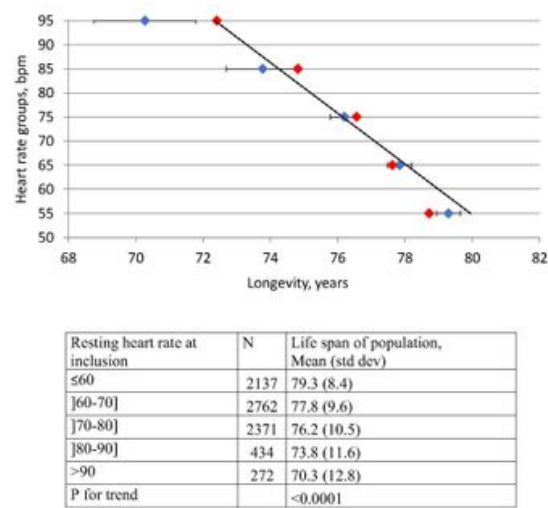

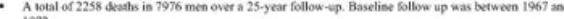

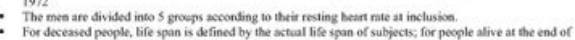

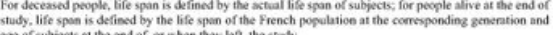

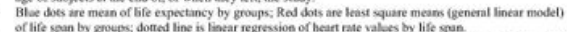

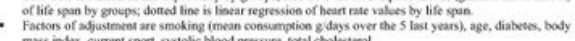

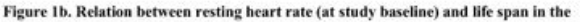
1226 men from the Whitehall I study
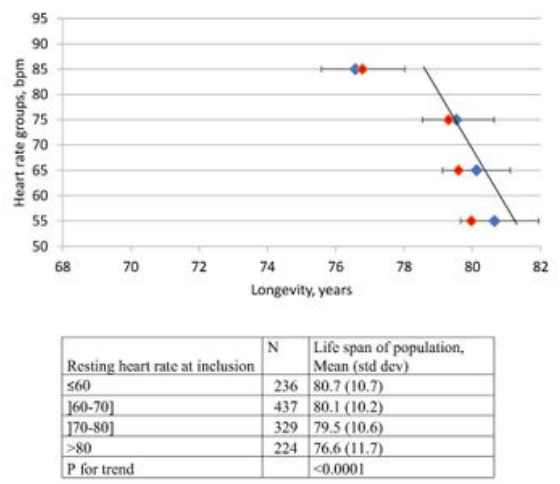

A total of 977 deaths ia 1226 persens over a 40 years follow-wp. Bascline follow up was at 196 ?

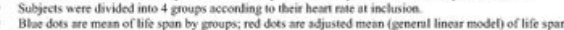

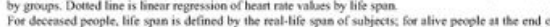

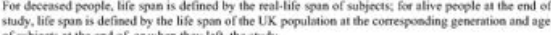

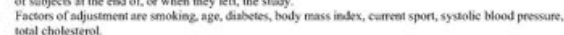

Figure Ic-ld. Relation between resting heart rate (at study baseline) and life span in 3299 men (e) and 4001 women (d) from the Framingham Original and Offispring cohorts
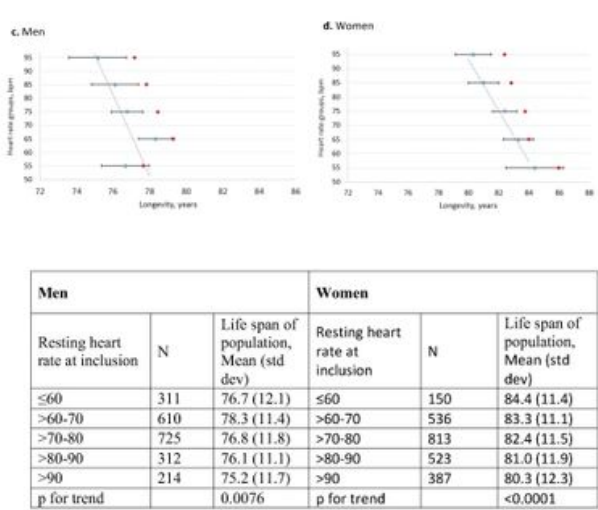

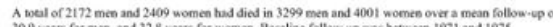

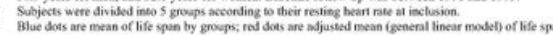

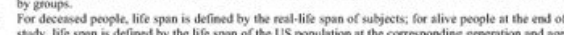
ing generation nand age

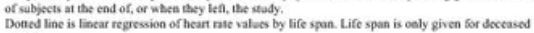

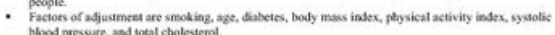

\section{Figure 1}

a. Relation between resting heart rate (at study baseline) and life span in 7967 men from the Paris Prospective study I

b. Relation between resting heart rate (at study baseline) and life span in the 1226 men from the Whitehall I study

c-d. Relation between resting heart rate (at study baseline) and life span in 3299 men (c) and 4001 women (d) from the Framingham Original and Offspring cohorts 
Figure 2. Relationship between heart rate and life span in mammals and historical data between 1750 and 2000 in men

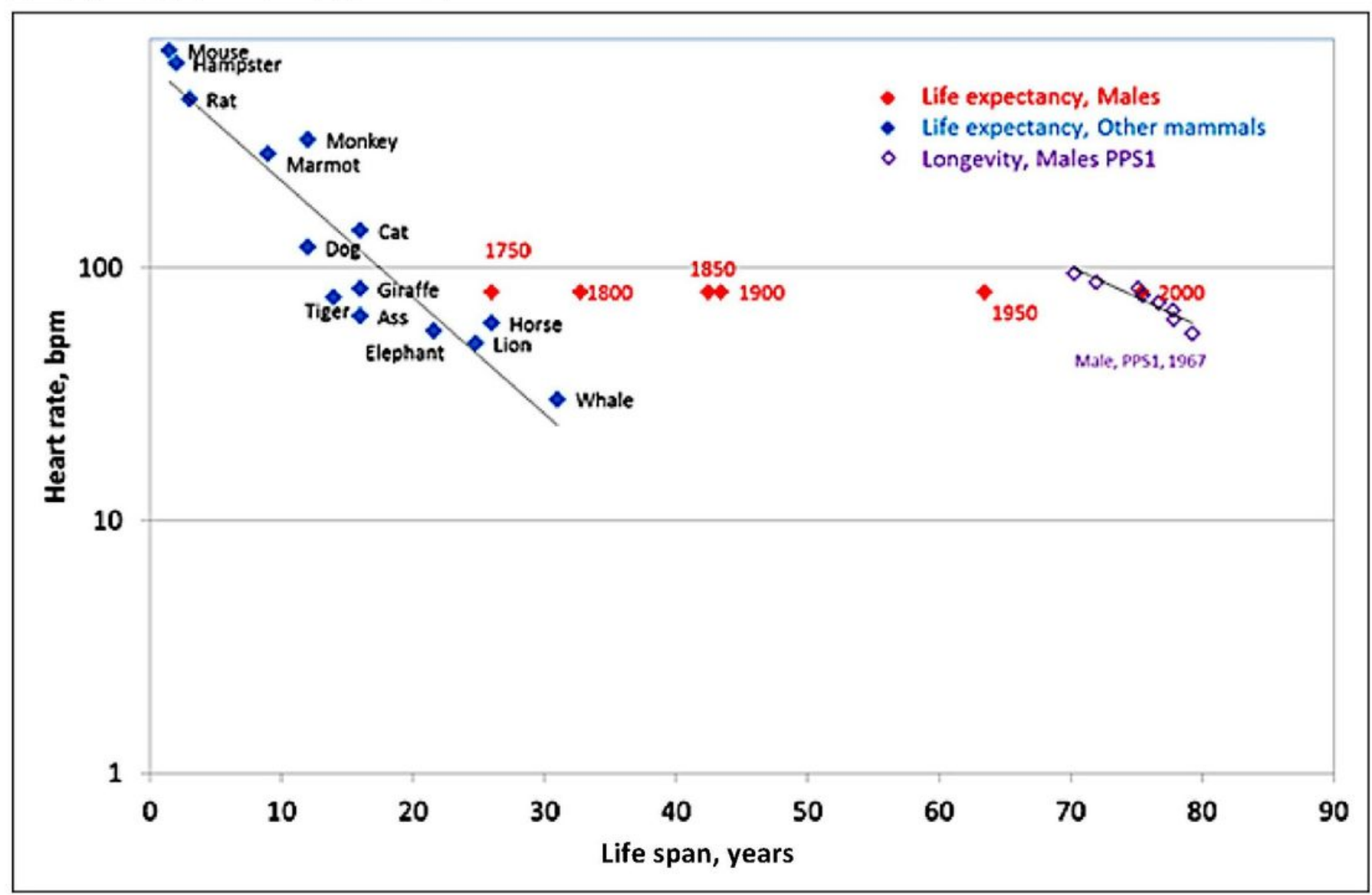

- Relation between logarithmic transformed heart rate and life span in mammalian species from Levine ${ }^{1}$

- Historical data (1750 to 2000) come from the French National Institute of Demographic Studies.

- Life span for French adults in 1750 , before the major advances in public health, sanitation, antisepsis and antibiosis, averaged 30 years, placed human life span near the otherwise predictable line of heart rate versus life span seen for other mammals.

Figure 2

Relationship between heart rate and life span in mammals and historical data between 1750 and 2000 in men 